\title{
Editorials
}

\section{Current equipment alarm sounds: friend or foe?}

Steven Dain MD FRCPC

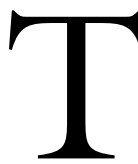

$\mathrm{HE}$ article on equipment alarm sounds in this issue by Mondor and Findlay ${ }^{1}$ partially confirms what every anesthesiologist and intensivist has suspected for years; that medical device alarm systems are often poorly designed and do not necessarily convey the appropriate sense of urgency. Others have found that current sounds are difficult to identify, startle and annoy, and have ambiguous modes of operation.

Mondor and Findlay examined numerous devices that are commonly found in any operating room (OR). They asked naïve and experienced persons to rate different equipment auditory alarm signals to their perceived sense of urgency. What they actually found was that, often, the perceived urgency of the alarm signal was not always congruent with the potential hazard and risk to the patient or healthcare provider.

Momtahan et al. ${ }^{2}$ studied the audibility and identification of alarms in the OR and intensive care unit (ICU). OR staff including nurses, anesthesia residents, and anesthesiologists were able to identify between ten and 15 of 23 alarms and the ICU nurses were able to identify nine to 14 of 23 different ICU alarms. An orthopedic drill completely masked 12 of the common anesthesia equipment alarm sounds. Using common cognitive criteria for pleasantness of alarm sounds, most of the alarms in this study would be considered unpleasant.

Similarly, Loeb et al. ${ }^{3}$ studied anesthesiologists' recognition accuracy of OR alarms. Clinicians correctly identified the alarm source only $34 \%$ of the time and Loeb concluded that anesthesiologists cannot reliably identify current OR alarms. Many of the alarms are spurious or false alarms due to patient movement, artifact, problems with the biological sensor, algorithms or the patient-equipment contact. One study, ${ }^{4}$ entitled "Crying wolf: false alarms in a pediatric ICU" con- cluded that over $94 \%$ of alarm sounds may not be clinically important.

In 1997, Tsien and Fackler, ${ }^{5}$ after studying 298 monitored hours in a pediatric ICU in which $86 \%$ of a total of 2,942 alarms were found to be false-positive alarms, declared a "poor prognosis for existing monitors in the ICU". However 64\% of these alarms were related to pulse oximetry. Recent improvements in probes and improved motion resistant algorithms within the newer crop of pulse oximeters should hopefully do much to improve this dismal statistic.

Alarm sounds may not be appropriate to the environment in which they are placed. Some are too loud, others not loud enough, others have a spectral content (fundamental frequency and harmonics) that make them difficult to hear within the patient environment or down the hall. Wallace et al. ${ }^{6}$ studied the hearing acuity of anesthesiologists and their ability to detect alarms. Subjects were divided into six groups according to age. Sixty-six percent of those tested had abnormal audiograms and median hearing threshold was worse than the general population for men and women under the age of 55 . In addition, older anesthesiologists had difficulty hearing some alarms with frequencies greater than $4000 \mathrm{~Hz}$ and missed some of them altogether; masked by the usual background noise in a busy OR.

As almost all devices today being microprocessor controlled, and as miniature microphones are an inexpensive commodity, it would not be expensive for manufacturers to monitor background noise and annunciate alarm signals $10 \mathrm{~dB}$ louder than the ambient noise levels; this is being done in some critical care ventilators today.

Not only are the alarm signals a problem. Alarm system controls may be ambiguous to use, modes of operation and alarm volumes difficult to determine;

From the Department of Anesthesia and Perioperative Medicine, University of Western Ontario, London, Ontario Canada.

Address correspondence to: Dr. Steven Dain, Department of Anesthesia and Perioperative Medicine, University of Western Ontario, London Health Sciences Centre, 339 Windermere Road, London, Ontario N6A 5A5, Canada. Phone: 519-663-3022; Fax: 519-6633161; E-mail: sdain@uwo.ca

Canadian Expert, ISO/IEC Joint Working Group on Medical Alarm Signals. 
TABLE Proposed equipment and priority encoded auditory alarm signals (letters represent musical notes)

\begin{tabular}{|c|c|c|c|c|}
\hline Cause & Medium priority & High priority & Mnemonic notes & Typical alarm system type \\
\hline General & $c \subset c$ & $c \mathrm{c}-\mathrm{c} c$ & Fixed pitch & $\begin{array}{l}\text { Other alarm systems that do not readily } \\
\text { fall into one of the following categories, } \\
\text { including but not limited to electrocardio- } \\
\text { gram, laparoscopy equipment, antiembolic } \\
\text { stockings, etc. }\end{array}$ \\
\hline Cardiac & $\mathrm{ce} \mathrm{g}$ & c e g-g C & $\begin{array}{l}\text { Trumpet call; call to } \\
\text { arms; major chord }\end{array}$ & $\begin{array}{l}\text { Anesthesia workstations that include cardiac } \\
\text { monitors, multi-parameter monitors which } \\
\text { include cardiac monitors, heart rate moni- } \\
\text { tors, invasive or noninvasive blood pressure } \\
\text { monitors, cardiac output monitors, peripher- } \\
\text { al perfusion monitors (plethysmographs), } \\
\text { transesophageal echo, fetal heart rate monitors. }\end{array}$ \\
\hline Artificial perfusion & $\mathrm{c}$ f\# c & $c \mathrm{f \#} \mathrm{c}-\mathrm{c} f \#$ & $\begin{array}{l}\text { Artificial sound; Tritone } \\
\text { "Yo-ee-oh" Munchkins } \\
\text { "The Wizard of Oz" }\end{array}$ & $\begin{array}{l}\text { Cardiopulmonary perfusion pumps ("heart- } \\
\text { lung machines") and associated equipment, } \\
\text { intra-aortic balloon pumps, renal } \\
\text { dialysis systems. }\end{array}$ \\
\hline Ventilation & $\mathrm{c}$ a f & $c$ a f-a f & $\begin{array}{l}\text { NBC Chime; inverted } \\
\text { major chord; rise and } \\
\text { fall of the lungs }\end{array}$ & $\begin{array}{l}\text { Anesthesia workstations that which included } \\
\text { artificial ventilators (but which do not } \\
\text { include cardiac monitors); artificial ventila- } \\
\text { tors, spirometers, } \mathrm{CO}_{2} \text { monitors, ventilator } \\
\text { disconnect (airway pressure) monitors, etc. }\end{array}$ \\
\hline Oxygen & $\mathrm{C} \mathrm{b}$ a & $\mathrm{C} \mathrm{b} a-g$ f & $\begin{array}{l}\text { Slowly falling pitches; } \\
\text { top of a major scale; } \\
\text { falling pitch of an oximeter }\end{array}$ & $\begin{array}{l}\text { Pulse oximeters, transcutaneous/tissue oxy- } \\
\text { gen monitors, oxygen analyzers, oxygen } \\
\text { concentrators, oxygen gas supply lines. }\end{array}$ \\
\hline Temperature/Energy delivery & $\mathrm{cd} \mathrm{e}$ & $c d e-f g$ & $\begin{array}{l}\text { Bottom of a major scale; } \\
\text { related to slow increase in } \\
\text { energy or (usually) } \\
\text { temperature slowly } \\
\text { rising pitches; }\end{array}$ & $\begin{array}{l}\text { Temperature monitors, heated air humidi- } \\
\text { fiers, infant radiant warmers, neonatal incu- } \\
\text { bators, patient heating or cooling systems, } \\
\text { blood or fluid warmers; electrocautery, ultra- } \\
\text { sound, laser, } x \text {-ray or magnetic resonance } \\
\text { imaging systems, nerve stimulators. }\end{array}$ \\
\hline Drug or fluid delivery & $\mathrm{C} \mathrm{d} \mathrm{g}$ & $\mathrm{C} \mathrm{d} \mathrm{g-C} \mathrm{d}$ & $\begin{array}{l}\text { Jazz chord (inverted } 9 \text { th); } \\
\text { drops of an infusion falling } \\
\text { and "splashing" back up }\end{array}$ & $\begin{array}{l}\text { Volumetric infusion pumps, syringe drivers, } \\
\text { anesthetic agent delivery systems or analyzers. }\end{array}$ \\
\hline Equipment or supply failure & $\mathrm{Ccc}$ & $\mathrm{C} c \mathrm{c}-\mathrm{C} c$ & & $\begin{array}{l}\text { Any device when it experiences loss of } \\
\text { power or other major failure of the device. }\end{array}$ \\
\hline Any low priority condition & e c & & & Any ding-dong hostess call or door bell \\
\hline
\end{tabular}

equipment, that from the outside appearance look the same, could have very different alarm settings, limits and modes of operation, of which the casual or even every day operator may not be aware. One particular monitor has a key on its front panel that can turn off an alarm system indefinitely or just for two minutes depending on how the device is programmed by the hospital biomedical engineering department. Injuries and death have resulted from ambiguous monitoring modes. ${ }^{7}$

The ideal medical device auditory and visual alarm system must not startle and annoy, must be loud enough to hear and of an appropriate spectral content such that it is heard around corners if necessary and not masked by the background noise; but not too loud or continuous in nature that it prevents easy communication between people. The auditory signals must be prioritized and convey the risk appropriate sense of urgency to the clinician. The controls, their labelling and configuration must be easy to use and non-ambiguous. Old ambiguous terms, such as "disable", "inhibit" and "suspend" must give way to the terms "audio off”, “audio paused”, “alarms off”, "alarms paused", more descriptive terms that are easily understood. More complex equipment, such as multiparameter monitors and critical care ventilators must have alarm condition and event logs similar to the "black boxes" on aircraft, to review brief alarm conditions and for the study and defense of critical incidents.

All equipment should have the means to communicate electronically its alarm state to other equipment, such as distributed medical equipment systems and information systems. Standards are still needed to accomplish this function. The Medical Information Bus (IEEE 1073) despite being in development for almost 20 years is still not a viable option for most devices. Hardware communications standards are required that are plug and play, have a unique con- 
nector, have error correction protocols and are sufficiently fast. Standardized XML (extensible mark-up language) schemas must be developed for every type of medical device, to allow the easy inexpensive movement of information between equipment so that expensive proprietary software "drivers" need not be written for each individual device. Manufacturers must no longer use the excuse that "we do not want to accept the liability and risk of letting another piece of equipment from another manufacturer annunciate our equipments' alarms". ${ }^{\text {A }}$ Manufacturers and hospital information systems providers must be cognizant that the Anesthesia Patient Safety Foundation (www.apsf.org) and the Database Task Force of the Canadian Anesthesiologists' Society are currently developing a standardized vocabulary for electronic anesthesia records, which may include alarm data.

It is my opinion that auditory alarm signals should be encoded as to the urgency of the problem and also encoded by type of device. In this way, all heart rate alarms, no matter which manufacturer has supplied the device, will sound the same.

The IEC/ISO joint working group on medical equipment alarm systems has proposed a new set of auditory alarm sounds which encode within the sound the source of the alarm condition. ${ }^{\mathrm{B}}$ It is proposed that there will be only eight different alarm sounds (Table). Psychological studies have shown that six to eight different sounds can be learned easily and recognized repeatedly. This will, hopefully, obviate the confusion over alarm signal recognition in areas with multiple devices.

Only through these means will clinicians have affordable ready access to fully integrated alarm systems that have the qualities of the ideal alarm system and more affordable computerized patient records.

\section{Alarmes sonores du matériel courant : ami ou ennemi ?}

Dans le présent numéro, l'article de Mondor et Findlay ${ }^{1}$ sur les sonneries d'alarme confirme ce que tous les anesthésiologistes et les intensivistes pensent depuis des années ; les systèmes d'alarme médicale sont souvent mal conçus et ne transmettent pas nécessairement le sens approprié de l'urgence. D'autres croient que les sonneries courantes sont difficiles à identifier, font sursauter ou dérangent, et présentent des modes de fonctionnement ambigus.

Mondor et Findlay ont vérifié de nombreux dispositifs qu'on retrouve fréquemment dans les salles d'opération $(\mathrm{SO})$. Ils ont demandé à des novices et à des personnes expérimentées d'évaluer les dispositifs d'alarmes sonores en fonction de l'urgence perçue. Ils ont trouvé que, souvent, l'urgence perçue ne concorde pas avec le danger et le risque potentiel pour le patient ou le prestateur de soins.

Momtahan et coll. ${ }^{2}$ ont étudié l'audibilité et l'identification des alarmes dans la $\mathrm{SO}$ et à l'unité des soins intensifs (USI). Le personnel de la SO, comprenant le personnel infirmier, les résidents en anesthésie et les anesthésiologistes, ont pu identifier de 10 à 15 des 23 alarmes et les infirmières de l'USI, de 9 à 14 parmi 23 alarmes différentes. Le bruit d'une fraise orthopédique masquait complètement 12 des alarmes sonores couramment utilisées en anesthésie. La plupart des alarmes de cette étude ont été jugées déplaisantes selon les critères cognitifs habituels.

De même, Loeb et coll. ${ }^{3}$ ont vérifié avec quelle précision les anesthésiologistes peuvent reconnaitre les alarmes de la SO. Les cliniciens ont correctement reconnu $34 \%$ seulement des sources d'alarme et Loeb en conclut qu'ils ne peuvent donc identifier fidèlement les alarmes courantes de la SO. De nombreuses alarmes parasites ou des fausses alarmes se déclenchent à cause de mouvements du patient, d'artéfact, de problèmes de détecteur biologique, d'algorithmes ou de contact matériel-patient. Une étude, ${ }^{4}$ intitulée Crying wolf: false alarms in a pediatric ICU (Crier au loup : fausses alarmes à l'USI pédiatrique), conclut que plus de $94 \%$ des sonneries d'alarmes peuvent être sans importance clinique.

En 1997, Tsien et Fackler, ${ }^{5}$ après avoir examiné 298 heures de monitorage à l'USI pédiatrique pen- 
TABLEAU Proposition d'alarmes sonores choisies en priorité pour divers types d'équipement

\begin{tabular}{|c|c|c|c|c|}
\hline Cause & Urgence moyenne & Urgence élevée & Notes mnémoniques & Systèmes d'alarme typiques \\
\hline Générale & do do do & do do do - do do & Tonalité fixe & $\begin{array}{l}\text { Autres systèmes d'alarme qui n'entrent pas } \\
\text { d'emblée dans l'une de ces catégories, y } \\
\text { compris, sans s'y limiter, l'électroencéphalo- } \\
\text { gramme, le matériel de laparoscopie, les bas } \\
\text { antiemboliques, etc. }\end{array}$ \\
\hline Cardiaque & do mi sol & do mi sol - sol DO & $\begin{array}{l}\text { Trompette ; appel aux } \\
\text { armes ; accord majeur }\end{array}$ & $\begin{array}{l}\text { Appareillage d'anesthésie incluant les moniteurs } \\
\text { cardiaques, les moniteurs multi-paramètres : les } \\
\text { moniteurs cardiaques, les moniteurs de la } \\
\text { fréquence cardiaque, les moniteurs effractifs ou } \\
\text { non de la tension artérielle, du débit cardiaque, } \\
\text { de la perfusion périphérique (pléthysmo- } \\
\text { graphes), du rythme cardiaque foetal et l'é } \\
\text { chographie transœsophagienne. }\end{array}$ \\
\hline Perfusion artificielle & do fa\# do & do fa\# do - do fa\# & $\begin{array}{l}\text { Son artificiel ; triple } \\
\text { tonalité "Yo-ee-oh" } \\
\text { Munchkins "Le magicien } \\
\text { d'Oz" }\end{array}$ & $\begin{array}{l}\text { Circulation extracorporelle (machines } \\
\text { coeur-poumon ) et matériel auxiliaire, ballon } \\
\text { intra-aortique, systèmes de dialyse rénale. }\end{array}$ \\
\hline Ventilation & do la fa & do la fa - la fa & $\begin{array}{l}\text { Clairon de NBC ; accord } \\
\text { majeur inversé ; } \\
\text { gonflement et } \\
\text { affaissement des poumons }\end{array}$ & $\begin{array}{l}\text { Appareillage d'anesthésie comprenant les } \\
\text { ventilateurs artificiels (excluant les moniteurs } \\
\text { cardiaques); spiromètres, moniteurs de } \mathrm{CO}_{2} \text {, } \\
\text { moniteurs de débranchement des ventilateurs } \\
\text { (basse pression), etc. }\end{array}$ \\
\hline Oxygène & DO si la & DO si la - sol fa & $\begin{array}{l}\text { Tonalités lentement } \\
\text { décroissantes ; partie } \\
\text { aiguë d'une gamme } \\
\text { majeure }\end{array}$ & $\begin{array}{l}\text { Oxymètres de pouls, moniteurs d'oxygène } \\
\text { transcutané/tissulaire, analyseurs d'oxygène, } \\
\text { concentrateurs d'oxygène, canalisations } \\
\text { d'alimentation en oxygène. }\end{array}$ \\
\hline $\begin{array}{l}\text { Température/ } \\
\text { Distribution } \\
\text { d'énergie }\end{array}$ & do ré mi & do ré mi -fa sol & $\begin{array}{l}\text { Sons graves d'une gamme } \\
\text { majeure ; liés à une lente } \\
\text { augmentation de l'énergie } \\
\text { ou (habituellement) de } \\
\text { la température; tonalités } \\
\text { en hausse lente }\end{array}$ & $\begin{array}{l}\text { Moniteurs de température, humidificateurs à } \\
\text { air chauffé, lits chauffants pour nouveau-nés, } \\
\text { incubateurs néonataux, couvertures chauffantes } \\
\text { ou refroidissantes, réchauffeurs de sang ou de } \\
\text { liquide ; systèmes d'électrocautérisation, } \\
\text { d'ultrasons, de laser, de radiographie ou } \\
\text { d'imagerie par résonance magnétique, } \\
\text { neurostimulateurs. }\end{array}$ \\
\hline $\begin{array}{l}\text { Administration } \\
\text { de médicament } \\
\text { ou de liquide }\end{array}$ & DO ré sol & DO ré sol - DO ré & $\begin{array}{l}\text { Accord de jazz }\left(9^{\mathrm{e}} \text { in }\right. \\
\text { versé }) \text {; gouttes à gouttes } \\
\text { d'une perfusion } \\
\text { refoulement avec } \\
\text { éclaboussures }\end{array}$ & $\begin{array}{l}\text { Pompes à perfusion volumétrique, pousse- } \\
\text { seringues, systèmes d'administration ou } \\
\text { analyseurs d'anesthésiques. }\end{array}$ \\
\hline $\begin{array}{l}\text { Défaillance du } \\
\text { matériel ou de } \\
\text { l'alimentation }\end{array}$ & Do do do & DO do do - DO do & & $\begin{array}{l}\text { Tout dispositif qui subit une panne de courant } \\
\text { ou une autre défaillance majeure. }\end{array}$ \\
\hline $\begin{array}{l}\text { Toute condition } \\
\text { de faible priorité }\end{array}$ & mi do & & & $\begin{array}{l}\text { Toute sonnerie d'appel ou sonnette } \\
\text { domestique «ding-dong» }\end{array}$ \\
\hline
\end{tabular}

dant lesquelles $86 \%$ de 2942 alarmes ont été jugées comme de faux-positifs, ont émis un "pronostic pauvre pour les moniteurs actuels de l'USI". Il reste que $64 \%$ de ces alarmes étaient reliées à l'oxymétrie pulsée. De récents perfectionnements des sondes et l'amélioration des algorithmes de résistance au mouvement pour la nouvelle génération d'oxymètres de pouls devraient, espérons-le, améliorer ces déplorables statistiques.
Les sonneries d'alarmes ne conviennent peut-être pas à l'environnement dans lequel elles sont placées. Certaines sont trop bruyantes, d'autres pas assez ; d'autres ont un contenu spectral (fréquence fondamentale et harmoniques), il peut donc être plus difficile de les entendre dans l'environnement du patient ou dans une salle. Wallace et coll. ${ }^{6}$ ont testé l'acuité auditive des anesthésiologistes et leur capacité à détecter les alarmes. Les sujets ont été répartis en six 
groupes d'âge. Soixante-six pour cent d'entre eux avaient un audiogramme anormal et le seuil auditif moyen était plus bas que celui de la population générale d'hommes et de femmes de moins de 55 ans. Ajoutons à cela que les plus âgés avaient des difficultés à entendre certaines alarmes dont les fréquences dépassaient les $4000 \mathrm{~Hz}$ et ne percevaient pas du tout d'autres alarmes masquées par le bruit ambiant habituel d'une SO en activité.

Presque tous les appareils étant aujourd'hui contrôlés par microprocesseurs et les microphones miniatures étant un accessoire bon marché, il n'en coûterait pas cher aux fabricants de contrôler le bruit environnant et de présenter des signaux d'alarme plus forts, de $10 \mathrm{~dB}$, que les niveaux de bruits ambiants; on le fait déjà pour certains ventilateurs des soins intensifs.

Les sonneries d'alarme ne sont pas le seul problème. Les commandes des systèmes d'alarme peuvent apparaitre ambiguës à l'usage, les modes de fonctionnement et les volumes d'alarme difficiles à déterminer ; des appareils, en apparence semblables, peuvent avoir des réglages d'alarme, des limites et des modes de fonctionnement différents que l'opérateur occasionnel ou même quotidien n'aurait pas notés. Un moniteur donné est muni d'une clé sur le panneau frontal qui permet de couper l'alarme pendant une période indéterminée ou pour deux minutes seulement selon la programmation réalisée par le service d'ingénierie biomédicale de l'hôpital. Des lésions et même des décès ont été causés par des ambiguïtés dans le fonctionnement des moniteurs. ${ }^{7}$

Le système d'alarme sonore et visuelle idéal en médecine ne doit pas faire sursauter ou déranger, doit être assez fort pour qu'on l'entende et avoir un contenu spectral approprié, perceptible à une certaine distance si nécessaire et non masqué par le bruit ambiant; mais pas trop fort ou continu de sorte qu'il nuise à la communication entre les gens. Les signaux sonores doivent être classés par ordre de priorité et transmettre au clinicien le sens de l'urgence en fonction du risque. Les réglages, leurs étiquettes et configurations doivent être faciles à utiliser et non ambigus. Les anciens termes imprécis, comme "désactiver", "invalider" et "arrêter" doivent être remplacés par "arrêt audio", "pause audio", "arrêt alarme", "pause alarme", termes plus descriptifs et facilement compris. Le matériel plus complexe, comme les moniteurs à paramètres multiples et les ventilateurs en soins intensifs, doit présenter des conditions d'alarme et des registres d'événements semblables à ceux des "boîtes noires" des avions qui permettent l'examen du résumé des conditions d'alarme et l'étude et la défense des incidents critiques.
Tout appareil devrait pouvoir communiquer électroniquement son état d'alarme à un autre appareil, tels des systèmes d'appareils médicaux distribués et des systèmes d'information. Des normes sont encore nécessaires pour accomplir cette fonction. Le bus d'information médicale (IEEE 1073), même si on y travaille depuis presque 20 ans, n'est pas encore une option viable pour la plupart des appareils. Il faut des normes de communications qui soient autoconfigurables, aient un connecteur unique et des protocoles de correction d'erreurs et soient suffisamment rapides. La syntaxe du XML normalisé (langage de balisage extensible) doit être élaborée pour chaque type de dispositif médical pour permettre la transmission facile et non coûteuse de l'information entre les appareils de sorte que les "programmes de gestion" d'un logiciel privé ne doivent pas être écrits pour chacun des appareils en particulier. Les fabricants ne doivent plus utiliser de prétexte disant : "nous ne voulons pas accepter la responsabilité et le risque qu'un appareil d'un autre fabricant présente les alarmes de nos équipements". " Les fabricants et les fournisseurs de systèmes d'information des hôpitaux doivent savoir que l'Anesthesia Patient Safety Foundation (Fondation pour la sécurité des patients en anesthésie) (www.apsf.org) et le Groupe de travail sur les bases de données de la Société canadienne des anesthésiologistes élaborent actuellement un vocabulaire normalisé des documents informatiques en anesthésie qui pourrait inclure des données sur les alarmes.

Nous croyons que les sonneries d'alarmes devraient être codées en fonction de l'urgence du problème et du type d'appareil. Ainsi, toutes les alarmes de fréquence cardiaque, peu importe le fabricant de l'appareil, auraient la même sonnerie.

Le groupe de travail mixte CEI/ISO sur les systèmes d'alarme médicaux a proposé un nouvel ensemble de signaux d'alarme qui encodent la source de l'état d'alarme à l'intérieur du signal. ${ }^{\mathrm{B}}$ On propose seulement huit sons différents (Tableau). Des études psychologiques ont montré que six à huit sons différents peuvent être facilement appris et reconnus de façon répétitive. Cette situation permettra, nous l'es-

A Quelques fabricants de matériel médical. Communication personnelle.

B CEI 60601-1-8. Appareils électromédicaux - Partie 1-8 : règles générales de sécurité - norme collatérale : systèmes d'alarme - règles, tests et recommandations règles générales et recommandations pour les systèmes d'alarme des appareils électromédicaux et des systèmes électromédicaux. Projet final de norme internationale, Genève, juin 2002. 
pérons, de dissiper la confusion autour de la reconnaissance des signaux d'alarme quand il y a de nombreux appareils en présence.

Ces moyens seront les seuls à permettre un accès abordable et facile à des systèmes d'alarme pleinement intégrés, qui ont les qualités de système d'alarme idéal, et à des dossiers de patients informatisés à meilleur coût.

\section{References}

1 Mondor T, Findlay A. Perceived urgency of auditory warning alarms used in the hospital operating room. Can J Anesth 2002: 221-28.

2 Momtahan K, Hetu R, Tansley B. Audibility and identification of auditory alarms in the operating room and intensive care unit. Ergonomics 1993; 36: 1159-76.

3 Loeb RG, Jones BR, Leonard RA, Behrman K. Recognition accuracy of current operating room alarms. Anesth Analg 1992; 75: 499-505.

4 Lawless ST. Crying wolf: false alarms in a pediatric intensive care unit. Crit Care Med 1994; 22: 981-5.

5 Tsien CL, Fackler JC. Poor prognosis for existing monitors in the intensive care unit. Crit Care Med 1997; 25: 614-9.

6 Wallace MS, Ashman MN, Matjasko MJ. Hearing acuity of anesthesiologists and alarm detection. Anesthesiology 1994; 81: 13-28.

7 FDA Manufacturer and User Facility Device Experience (MAUDE) Database http://www.accessdata.fda.gov/scripts/cdrh/cfdocs/cfMAUDE/search.cfm 